\title{
Antimicrobial effects of a microemulsion and a nanoemulsion on enteric and other pathogens and biofilms
}

\author{
Paula C. Teixeira ${ }^{a, *}$, Gonçalo M. Leite ${ }^{a}$, Ricardo J. Domingues ${ }^{a}$, \\ Joana Silva ${ }^{a}$, Paul A. Gibbs ${ }^{\text {a,b }}$, João Paulo Ferreira ${ }^{a}$ \\ ${ }^{a}$ Escola Superior de Biotecnologia, R. Dr. António Bernardino de Almeida, 4200-072 Porto, Portugal \\ ${ }^{\mathrm{b}}$ Leatherhead Food International, Randalls Road, Leatherhead, Surrey KT22 7RY, UK
}

\begin{abstract}
Some microemulsions and nanoemulsions may have antimicrobial properties and be effective anti-biofilm agents. We examined the abilities of two fine emulsions, designated BCTP and TEOP, to inactivate suspensions of vegetative cells of Salmonella spp. Escherichia coli 0157:H7 (VT-), Pseudomonas aeruginosa, Staphylococcus aureus and Listeria monocytogenes. BCTP is an O/W nanoemulsion of soybean oil and tri-n-butyl phosphate emulsified with Triton X-100, while TEOP is an O/W microemulsion of ethyl oleate with Tween 80 as emulsifier and $n$-pentanol as a co-emulsifier. BCTP was effective in reducing the cell numbers of L. monocytogenes, while TEOP was effective against all five organisms investigated. The abilities of these emulsions to reduce preformed biofilms of the five bacteria were also investigated. With the exception of the biofilm formed by L. monocytogenes, which surprisingly was not significantly affected by BCTP, all biofilms were inhibited by both BCTP and TEOP.
\end{abstract}

Keywords: Microemulsions; Nanoemulsions; Antimicrobial effect; Biofilms; Foodborne pathogens

\section{Introduction}

Current methods of sanitation of food facilities have some well-known drawbacks, such as toxicity of cleaning agents' residues, or promotion of resistance to these agents in enteric pathogens. Therefore, there is a strong requirement to develop alternative sanitation and disinfection methods.

A particularly challenging problem in many environments, including food processing areas, is the presence of biofilms. These accumulations of inorganic and organic materials and bacteria can develop on most types of surfaces. Both pathogenic and non-pathogenic microorganisms can be incorporated into biofilms during their progressive formation. Although biofilms can become strongly attached to surfaces, parts of the biofilm can occasionally slough off to contaminate other surfaces as well as food products, when in a food processing environment. Biofilms that contain Listeria, Pseudomonas, Campylobacter, Escherichia coli or Salmonella all have been observed in food processing environments (Joseph et al., 2001). Biofilms are highly prevalent and difficult to remove and bacteria in them have enhanced resistance to antimicrobial agents and sanitizers (Burmolle et al., 2006).

In recent years, two research groups (mentioned below) have shown that some microemulsions and nanoemulsions have antimicrobial properties. The structures and physical properties of fine emulsions can be quite variable, giving rise to different nomenclature in the literature. The ones we will focus on are oilin-water $(\mathrm{O} / \mathrm{W})$ emulsions of the micellar type. True microemulsions are thermodynamically stable systems and are transparent or translucent. Nanoemulsions are only kinetically stable, and mostly opaque (Lawrence and Rees, 2000).

A soybean oil based nanoemulsion, for which extensive data have been reported, has been shown to have bactericidal properties against Gram-positive but not against enteric Gramnegative species (Hamouda and Baker, 2000); it is sporicidal, in

\footnotetext{
* Corresponding author. Fax: +351 225580095.

E-mail address: pcteixeira@esb.ucp.pt (P.C. Teixeira).
} 
dilutions up to 1:1000 (Hamouda et al., 1999, 2001); it has antiviral properties, but only on enveloped viruses (Donovan et al., 2001; Hamouda et al., 2001) and it is fungistatic, but not fungicidal (Hamouda et al., 2001). The focus of these studies has been on human health applications.

Another research group worked with a true $\mathrm{O} / \mathrm{W}$ microemulsion of ethyl oleate emulsified by Tween 80 and $n$ pentanol. They showed that the emulsion gave a $5 \log$ reduction in the numbers of Staphylococcus aureus or Pseudomonas aeruginosa in only $45 \mathrm{~s}$, and a $90 \%$ reduction of the original microbial population in $15 \mathrm{~s}$ (Al-Adham et al., 2000). Later, the same group showed that the emulsion is active against biofilms of $P$. aeruginosa (Al-Adham et al., 2003).

In the studies reported here, we corroborated some of the previous observations, and extended the investigations to other pathogens, particularly the ones more commonly found in food items and on food processing equipment. We analysed the kinetics of inactivation of planktonic cultures of various microorganisms by these emulsions used at various dilutions. We also tested their capacity to destroy biofilms formed by different pathogens.

\section{Materials and methods}

\section{Bacterial strains and growth conditions}

S. aureus NCTC 1803, Salmonella typhimurium PSB 367, L. monocytogenes, P. aeruginosa, and E. coli O157:H7 (VT negative) were generously supplied by the culture collection of Escola Superior de Biotecnologia, Porto, Portugal. Bacterial cultures were maintained at $-80{ }^{\circ} \mathrm{C}$ in tryptone soy broth (TSB) (LabM, Bury, UK) with $30 \% \mathrm{v} / \mathrm{v}$ glycerol. Working cultures were grown on tryptone soy agar (TSA). TSB was inoculated with colonies from TSA plates and incubated at $37^{\circ} \mathrm{C}$ for $24 \mathrm{~h}$. This culture was then used to inoculate fresh TSB. For biofilm formation, the media used were: TSB for $P$. aeruginosa (Henriques et al., 2005); 1/20 TSB for S. typhimurium (Stepanović et al., 2004) and E. coli (Mathur et al., 2006); and Brain Heart Infusion (BHI) broth (LabM) for S. aureus (Ryu et al., 2004) and L. monocytogenes (Stepanović et al., 2004).

\section{Preparation of emulsions}

The BCTP nanoemulsion was prepared according to Hamouda et al. (1999) and Baker et al. (2003). The TEOP microemulsion was prepared according to Al-Adham et al. (2000). The oil-in-water $(\mathrm{O} / \mathrm{W})$ nanoemulsion, has an oil phase of soybean oil $(16 \% \mathrm{v} / \mathrm{v}$ of the total emulsion), tri- $n$-butyl phosphate $(2 \%)$, and triton X-100 (2\%). Therefore, this phase represents $20 \%(\mathrm{v} / \mathrm{v})$ of the emulsion. The components of the oil phase were mixed and kept for $1 \mathrm{~h}$ at $86^{\circ} \mathrm{C}$. After this, the water phase was added, and the mixture was emulsified with a high sheer laboratory mixer (Ultra-Turrax T25, Janke \& Kunkel GMBH, Staufen, Germany) for $5 \mathrm{~min}$. The microemulsion is also an $\mathrm{O} / \mathrm{W}$ type, with the oil phase comprised of ethyl oleate $(3 \% \mathrm{v} / \mathrm{v}$ of the total emulsion), $n$-pentanol $(6 \%)$, and tween 80
$(15 \%)$. After adding the water to the oil phase, emulsification was promoted by vortex mixing.

\section{Kinetics of killing}

Overnight bacterial cultures were added at $1 \% \mathrm{v} / \mathrm{v}$ to undiluted and to ten- and hundred-fold dilutions of the emulsions. After the addition of each culture and at 1, 5, 15 and $30 \mathrm{~min}, 1 \mathrm{ml}$ samples were taken from those inoculated emulsions and immediately diluted in $9 \mathrm{ml}$ of Ringer's solution at room temperature. For viable counts, samples were spread plated onto TSA and the plates were incubated at $37^{\circ} \mathrm{C}$ for $24 \mathrm{~h}$. At least two independent experiments were carried out for each set of conditions.

\section{Biofilm studies}

Biofilm formation in plastic microplates was performed as previously described by Stepanović et al. (2004). A $20 \mu \mathrm{l}$ portion of an overnight broth culture was added to each well of a 24-well tissue culture plate (Sarstedt, Newton, NC, USA) which was incubated aerobically, with mild agitation at $70 \mathrm{rpm}$, for $72 \mathrm{~h}$ at $37^{\circ} \mathrm{C}$. Every $12 \mathrm{~h}$, the medium containing suspended bacterial cells was removed and an equal volume of fresh medium was added. Negative controls were obtained by incubating the microplates with media without inocula. At least three independent experiments were performed.

After removing the supernatant media, the biofilms were treated with $2.5 \mathrm{ml}$ of each emulsion, for 5 and $30 \mathrm{~min}$ (in separate wells), at room temperature and without agitation. After that, the emulsion was removed and the wells were gently washed twice with sterilized distilled water.

Quantification of viable cells in biofilms in plastic microplates was performed as previously described (Stepanović et al., 2004). Attached bacteria were fixed with $2.5 \mathrm{ml}$ of methanol per well, for $15 \mathrm{~min}$. The microplates were then emptied and air dried. Each well was then stained for $5 \mathrm{~min}$ with $2.5 \mathrm{ml}$ of $1 \%(\mathrm{v} / \mathrm{v})$ crystal violet. Excess stain was rinsed off by placing the microplate under running tap water. The microplates were air dried and the dye that bound to the adherent cells was removed with $5 \mathrm{ml}$ of $33 \%(\mathrm{v} / \mathrm{v})$ glacial acetic acid per well. The optical density of the resulting solutions was read at $595 \mathrm{~nm}$ on a microplate reader (Model 3550, BioRad, Hercules, CA, USA).

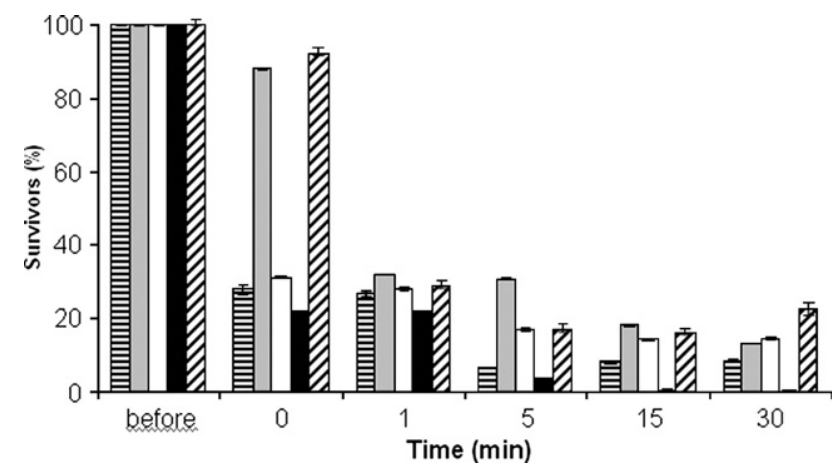

Fig. 1. Effect of the BCTP nanoemulsion on the survival of Salmonella typhimurium (目), Escherichia coli $(\square)$, Pseudomonas aeruginosa $(\square)$, Staphylococcus aureus $(\square)$ and Listeria monocytogenes ( $\square)$. 


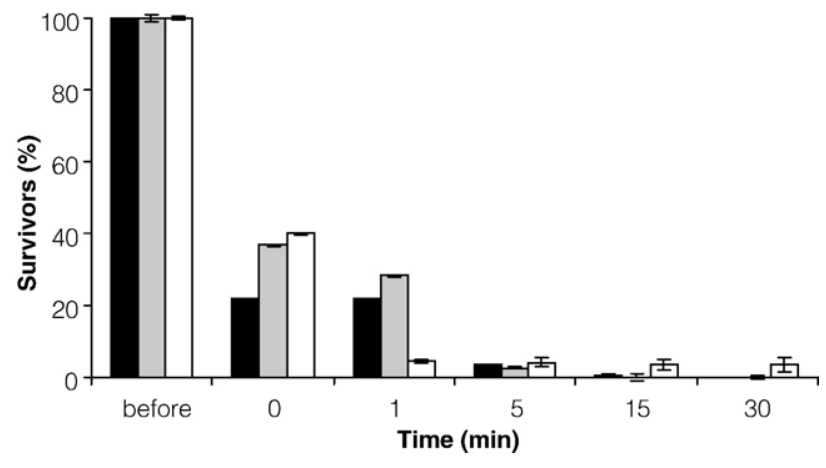

Fig. 2. Effect of the BCTP nanoemulsion, undiluted ( $\mathbf{\square})$, and diluted 1/10 BCTP $(\square)$ and 1/100 BCTP ( $\square$ ) on the survival of Listeria monocytogenes.

In each case, the mean optical density of the negative control was subtracted from the readings of all other samples.

\section{Results and discussion}

Fine emulsions have long been employed in some applications, such as in detergents (Azemar, 1997), pharmaceuticals (Tenjarla, 1999; Lawrence and Rees, 2000) and cosmetics (Sonneville-Aubrun et al., 2004). The use of some fine emulsions as antimicrobials is a quite new and promising application. However, there is limited number of reports in the literature on their use for this purpose. Furthermore, there is a pressing need to develop novel strategies to combat the continuous increase in microbial resistance, due to the widespread, and sometimes inappropriate, use of antibiotics, disinfectants and antiseptics.

\section{Kinetics of killing}

During the tested period of incubation with cell suspensions, BCTP reduced the number of survivors of all the pathogenic microorganisms (Fig. 1). BCTP reduced the fraction of survivors by at least $70 \%$ in just $1 \mathrm{~min}$. After that, the reduction was more pronounced for L. monocytogenes, with no survivors of this microorganism remaining after $15 \mathrm{~min}$ of incubation.
Fig. 2 shows the dependency of the kinetics of inactivation of L. monocytogenes upon the dilution of BCTP. After the addition of this agent, at time 0 , and after $1 \mathrm{~min}$, differences between the numbers of bacteria were found. However, after $5 \mathrm{~min}$ and later, ten-fold and 100-fold diluted BCTP were equally effective against this pathogen. Given this, with regard to L. monocytogenes, BCTP was shown to be a potential alternative "sanitizer" for the food sector. To our knowledge, this was the first time that this or any other emulsion was evaluated as an antimicrobial for reducing or eliminating L. monocytogenes. This organism is responsible for listeriosis, a severe infection among the elderly, the very young and the immunocompromised individuals. Listeriosis has also been associated with lateterm miscarriages in pregnant woman (Almeida et al., 2006). As for the other pathogens that were tested, and in agreement with the data of Baker et al. (2003), BCTP could reduce, but not completely eliminate them. Our results are also in agreement with other previous findings that BCTP had bactericidal properties against Gram-positive but not against enteric Gramnegative species (Hamouda et al., 2001; Hamouda and Baker, 2000). BCTP has been described as a non-toxic nanoemulsion (Hamouda et al., 2001; Chepurnov et al., 2003). However, Baker et al. (2003) reported that the undiluted form of this agent promoted necrosis in CD-1 type mice.

In contrast, TEOP was found to be active against all the tested pathogens. In fact, cells treated with TEOP showed an almost complete loss of viability immediately after their addition to the microemulsion. That is, given the sensitivity of the counting technique, the numbers were less than $100 \mathrm{CFU} / \mathrm{ml}$ immediately after the addition of the cultures to the emulsion, which corresponds to reductions in numbers of more than $99 \%$ (data not shown). These results are in agreement with those previously reported by Al-Adham et al. (2000) for P. aeruginosa and S. aureus.

Curiously, TEOP lost all antimicrobial properties when diluted 10-fold and 100-fold. As TEOP is a true microemulsion its structure is probably quite sensitive to compositional changes. Therefore, it is likely that the addition of water leads to significant changes in structure that affect its antimicrobial

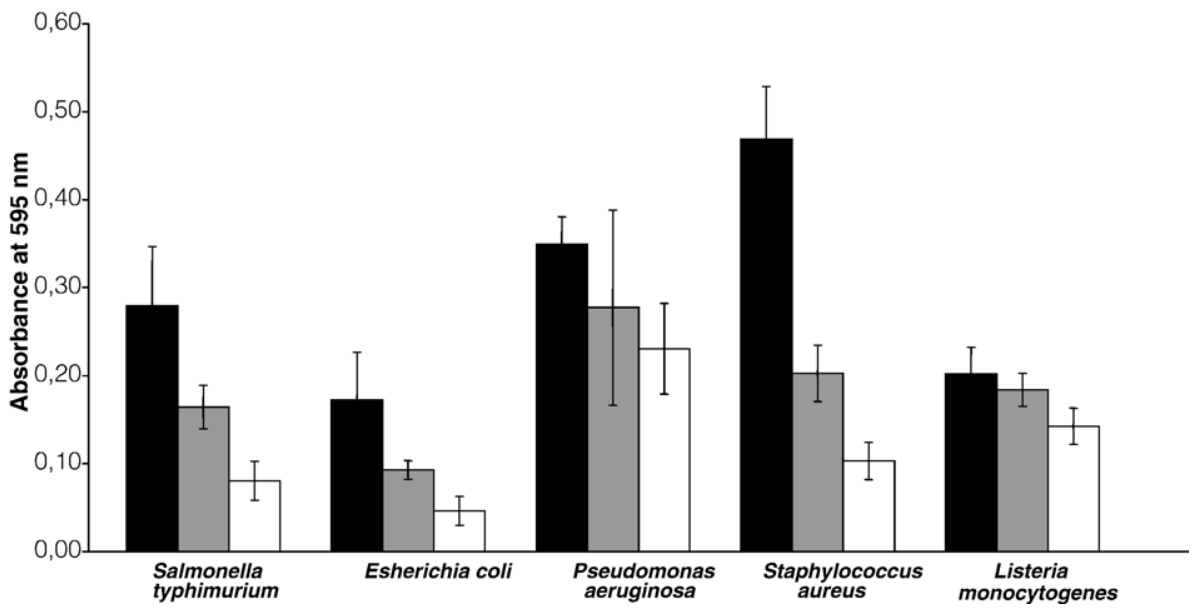

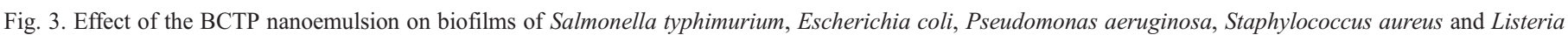
monocytogenes at $0 \mathrm{~min}(\square), 5 \mathrm{~min} .(\square)$ and $30 \mathrm{~min}(\square)$. 


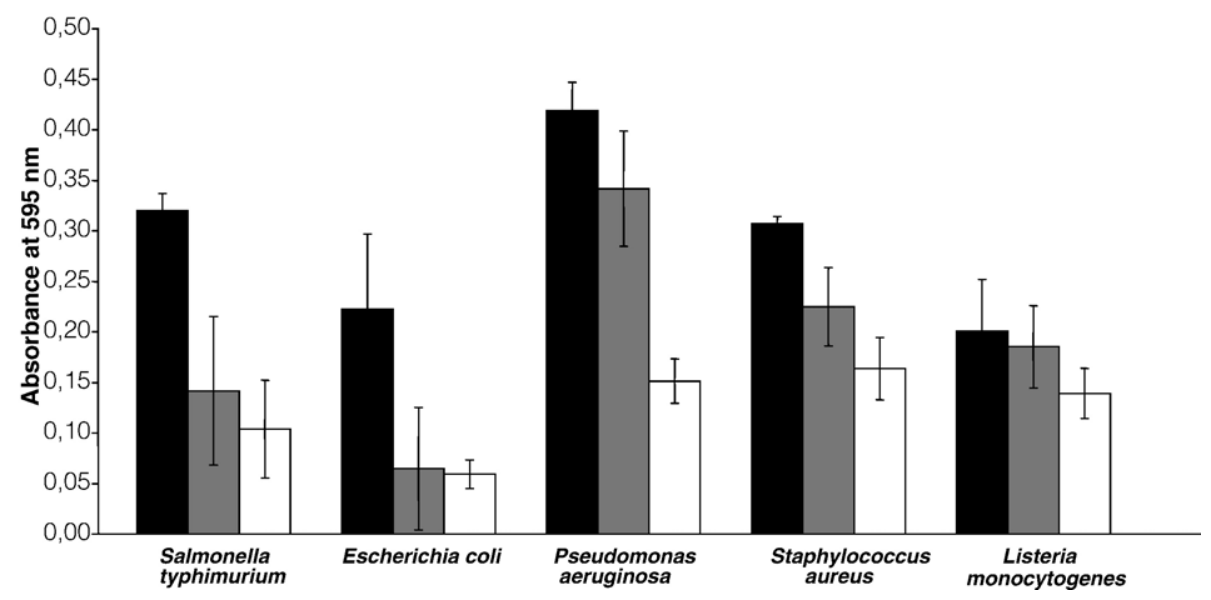

Fig. 4. Effect of the TEOP microemulsion on biofilms of Salmonella typhimurium, Escherichia coli, Pseudomonas. aeruginosa, Staphylococcus aureus and Listeria monocytogenes at $0 \mathrm{~min}(\square), 5 \mathrm{~min}$. ( $\square$ ) and $30 \mathrm{~min}(\square)$.

properties. In fact, recent work has analysed the relationship between antimicrobial activity and the position of the microemulsion within its zone of stability (Al-Navajeh, 2003).

\section{Biofilm studies}

The formation of biofilms by the tested pathogens on microplates was previously optimized (data not shown). It was observed that the maximum quantities of biofilms were produced $72 \mathrm{~h}$ after seeding. These biofilms were formed during fed-batch cultivation. This is different from most other studies, in which the biofilms were formed during batch cultivation for one day. This latter method does not approximate the real situations in either clinical or food processing environments (Cerca et al., 2004).

The capacities of BCTP and TEOP to remove the biofilms of the selected pathogens were evaluated. Generally, with both emulsions, $30 \mathrm{~min}$ exposure gave the maximum reductions of biofilms. BCTP showed higher activity against the biofilms formed by S. typhimurium, E. coli O157:H7 (VT-) and S. aureus than against those formed by $P$. aeruginosa or L. monocytogenes (Fig. 3). P. aeruginosa is known to be a nosocomial agent that is very difficult to eliminate when forming biofilms on different materials (Defez et al., 2004). With regard to L. monocytogenes, it was surprising to observe that BCTP, which had effectively reduced the numbers of the planktonic form, did not eliminate these cells in a biofilm. This might be due to a low exposure time or, more likely, to a particularly resistant biofilm. Further work should be carried out to investigate the mechanisms of the interactions between emulsions, cells in suspension and biofilms. This is the first reported evaluation of the action of BCTP on biofilms.

TEOP reduced the biofilms produced by $P$. aeruginosa, E. coli O157:H7 (VT-), S. typhimurium and S. aureus (Fig. 4). AlAdham et al. (2003) also found that this microemulsion was highly effective against biofilms of $P$. aeruginosa and $S$. aureus. However, the biofilm produced by $L$. monocytogenes was resistant to this emulsion as well. This is in agreement with previous reports that this bacterium produces biofilms, in many different environments, in which the cells are very resistant to cleaning agents (Chmielewski and Frank, 2003; Møretrø and Langsrud, 2004). The reasons for the higher resistance of L. monocytogenes biofilms to fine emulsion as well as to other antimicrobial agents require further investigation.

\section{References}

Al-Adham, I.S.I., Khalil, E., Al-Hmoud, N.D., Kierans, M., Collier, P.J., 2000. Microemulsions are membrane-active, antimicrobial, self-preserving systems. Journal of Applied Microbiology 89, 32-39.

Al-Adham, I.S.I., Al-Hmoud, N.D., Khalil, E., Kierans, M., Collier, P.J., 2003. Microemulsions are highly effective anti-biofilm agents. Applied Microbiology 36, 97-100.

Almeida, G., Gibbs, P., Hogg, T., Teixeira, P., 2006. Listeriosis in Portugal: an existing but unreported infection. BMC Infectious Diseases 6, 153 (http:// www.biomedcentral.com/1471-2334/6/153).

Al-Navajeh, A.S.M., 2003. Studies on the Microbiology of Oil-in-Water Microemulsions. PhD thesis, University of Abertay, Dundee, UK.

Azemar, N., 1997. The role of microemulsions in detergency processes. In: Solans, C., Kunieda, H. (Eds.), Industrial Applications of Microemulsions. Marcel Dekker, New York, pp. 375-388.

Baker Jr., J.R., Hamouda, T., Shih, A., Andrzej, M., 2003. Non-toxic antimicrobial compositions and methods of use. US Patent 6,559,189.

Burmolle, M., Webb, J.S., Rao, D., Hansen, L.H., Sorensen, S.J., Kjelleberg, S., 2006. Enhanced biofilm formation and increased resistance to antimicrobial agents and bacterial invasion are caused by synergistic interactions in multispecies biofilms. Applied and Environmental Microbiology 72, 3916-3923.

Cerca, N., Pier, G.B., Vilanova, M., Oliveira, R., Azeredo, J., 2004. Influence of batch or fed-batch growth on Staphylococcus epidermidis biofilm formation. Applied Microbiology 39, 420-424.

Chepurnov, A.A., Bakulina, L.F., Dadaeva, A.A., Ustinova, E.N., Chepurnova, T.S., Baker Jr., J.R., 2003. Inactivation of Ebola virus with a surfactant nanoemulsion. Acta Tropica 87, 315-320.

Chmielewski, R.A.N., Frank, J.F., 2003. Biofilm formation and control in food processing facilities. Comprehensive Reviews in Food Science and Food Safety 2, 22-33.

Defez, C., Fabbro-Perav, P., Bouziges, N., Gouby, A., Mahamat, A., Daures, J.P., Sotto, A., 2004. Risk factors for multidrug-resistant Pseudomonas aeruginosa nosocomial infection. Journal of Hospital Infection 57, 209-216.

Donovan, B.W., Reuter, J.D., Cao, Z., Myc, A., Johnson, K.J., Baker Jr., J.R., 2001. Prevention of murine influenza A virus pneumonitis by surfactant nano-emulsions. Antiviral Chemistry and Chemotherapy 11, 41-49. 
Hamouda, T., Baker Jr., J.R., 2000. Antimicrobial mechanism of action of surfactant lipid preparations in enteric Gram-negative bacilli. Journal of Applied Microbiology 89, 397-403.

Hamouda, T., Hayes, M.M., Cao, Z., Tonda, R., Johnson, K., Wright, D.C., Brisker, J., Baker, J.R., 1999. A novel surfactant nanoemulsion with broadspectrum sporicidal activity against Bacillus species. The Journal of Infectious Diseases 180, 1939-1949.

Hamouda, T., Myc, A., Donovan, B., Shih, A.Y., Reuter, J.D., Baker Jr., J.R., 2001. A novel surfactant nanoemulsion with a unique non-irritant topical antimicrobial activity against bacteria, enveloped viruses and fungi. Microbiological Research 156, 1-7.

Henriques, M., Sousa, C., Lira, M., Elisabete, M., Oliveira, R., Oliveira, R., Azeredo, J., 2005. Adhesion of Pseudomonas aeruginosa and Staphylococcus epidermidis to silicone-hydrogel contact lenses. Optometry and Vision Science 82, 446-450.

Joseph, B., Otta, S.K., Karunasagar, I., Karunasagar, I., 2001. Biofilm formation by Salmonella spp. on food contact surfaces and their sensitivity to sanitizers. International Journal of Food Microbiology 64, 367-372.

Lawrence, M.J., Rees, G.D., 2000. Microemulsion-based media as novel drug delivery systems. Advanced Drug Delivery Reviews 45, 89-121.
Mathur, T., Singhal, S., Khan, S., Upadhyay, D.J., Fatma, T., Rattan, A., 2006. Detection of biofilm formation among the clinical isolates of Staphylococci: an evaluation of three different screening methods. Indian Journal of Medical Microbiology 24, 25-29.

Møretrø, T., Langsrud, S., 2004. Listeria monocytogenes: biofilm formation and persistence in food processing environments. Biofilms 1, 107-121.

Ryu, J.H., Kim, H., Beuchat, L.R., 2004. Attachment and biofilm formation by Escherichia coli $\mathrm{O} 157: \mathrm{H7}$ on stainless steel as affected by exopolysaccharide production, nutrient availability, and temperature. Food Microbiology, IFT Annual Meeting, July 12-16 — Las Vegas, NV.

Sonneville-Aubrun, O., Simonnet, J.-T., L'Alloret, F., 2004. Nanoemulsions: a new vehicle for skincare products. Advances in Colloid and Interface Science 108-109, 145-149.

Stepanović, S., Ćirković, I., Ranin, L., Švabić-Vlahović, M., 2004. Biofilm formation by Salmonella spp. and Listeria monocytogenes on plastic surface. Letters in Applied Microbiology 38, 428-432.

Tenjarla, S., 1999. Microemulsions: an overview and pharmaceutical applications. Critical Reviews in Therapeutic Drug Carrier Systems 16, 461-521. 